\title{
Effects of Antipsychotics on Arrhythmogenic Parameters in Schizophrenia Patients: Beyond Corrected QT Interval
}

This article was published in the following Dove Press journal: Neuropsychiatric Disease and Treatment

\author{
Hiroaki Okayasu (D) \\ Takahiro Shinozaki' \\ Yumiko Takano' \\ Norio Sugawara $\mathbb{D}^{\prime}$ \\ Kumiko Fujii ${ }^{2}$ \\ Norio Yasui-Furukori (D) \\ Yuji Ozeki \\ Kazutaka Shimoda' \\ 'Department of Psychiatry, Dokkyo \\ Medical University, Tochigi, Japan; \\ ${ }^{2}$ Department of Psychiatry, Shiga \\ University of Medical Science, Shiga, Japan
}

Purpose: Antipsychotic drugs have been implicated as risk factors for QT prolongation, which is a predictor of sudden cardiac death. However, the QT interval is considered an imperfect marker for proarrhythmic risk. Recently, improved methods, namely, QT dispersion (QTD), QTD ratio (QTDR), T wave peak-to-end interval (Tp-e), Tp-e/QT ratio and Tpe/QTc ratio, have been regarded as proarrhythmic risk markers. We attempted to reevaluate the risk of sudden cardiac death due to antipsychotics use by measuring these improved evaluation methods.

Patients and Methods: We retrospectively evaluated QTc, QTD, QTDR, Tp-e, Tp-e/QT ratio and $\mathrm{Tp}$-e/QTc ratio from the medical records of 410 patients with schizophrenia diagnosed by the Diagnostic and Statistical Manual of Mental Disorders, 4th Edition, Text Revision, or 5th Edition. Information on drugs administered was obtained from medical records. We investigated the correlation between each index on ECG and medication, such as antipsychotics, prescribed to participants with linear regression analysis. We also compared each index between 235 healthy controls and 235 patients matched for age and sex.

Results: Positive correlations between QTc and levomepromazine and brexpiprazole were identified. Levomepromazine and lithium were positively correlated with QTD. Levomepromazine, quetiapine, asenapine, clozapine and carbamazepine were positively correlated with QTDR. Levomepromazine, olanzapine, brexpiprazole and lithium were positively correlated with Tp-e. Olanzapine, brexpiprazole and lithium were positively correlated with the Tp-e/QT ratio. Olanzapine, brexpiprazole and lithium were positively correlated with Tp-e/QTc ratio. Significant differences in all indexes were noted between the patients and healthy controls.

Conclusion: According to our results, the prediction of the risk of sudden cardiac death by each index was inconsistent. We should evaluate the predictive factor of ventricular arrhythmia according to various electrocardiogram indexes because QTc alone could not identify the risk of sudden cardiac death.

Keywords: antipsychotic drugs, sudden cardiac death, electrocardiogram, QT prolongation, QT dispersion, T peak-to-end interval

\section{Introduction}

Schizophrenia is associated with a life loss of approximately 15 years. ${ }^{1}$ Cardiovascular disease was identified as the most common cause of premature death in patients with schizophrenia in a cohort study. ${ }^{2}$ An increased incidence of sudden cardiac death has been reported among patients treated with antipsychotic drugs. ${ }^{3}$
Correspondence: Hiroaki Okayasu

Tel +8I 282-86-IIII

Email hokayasu@dokkyomed.ac.jp

Neuropsychiatric Disease and Treatment 2021:17 239-249 
A prolonged QT interval on electrocardiography (ECG) is a risk factor for torsade de pointes, which can be a cause of sudden cardiac death. ${ }^{4}$ Because the QT interval value depends on heart rate, this value has been corrected to the RR interval, which means heart rate as corrected QT (QTc) in general ${ }^{5}$. It has been reported that antipsychotic drugs cause corrected QTc lengthening in a dose-related manner. ${ }^{6}$ We reported that administration of antipsychotic doses at greater than 1000 and $1500 \mathrm{mg} /$ day of chlorpromazine $(\mathrm{CP})$ equivalent increases the risk of QTc prolongation, ${ }^{7}$ and chlorpromazine, intravenous haloperidol and sultopride were associated with an increased risk of QTc prolongation, whereas secondgeneration antipsychotic drugs did not prolong the QTc interval. $^{7}$ However, the QT interval is recognized as an imperfect biomarker of proarrhythmic risk. ${ }^{8}$ The QT interval varies according to the ECG context. Continuous measurements of the QT interval from 24-hour ECG recordings reveal QT interval instability. ${ }^{9}$ Furthermore, the QT interval is prolonged during sleep. ${ }^{10}$ Hence, the estimation of other more precise indexes is needed to correctly stratify the risk factors for sudden cardiac death related to antipsychotic drug medication.

Several ECG markers, such as QT dispersion (QTD), QTD ratio (QTDR), T peak-to-end interval (Tp-e), Tp-e/ QT ratio and Tp-e/QTc ratio, are considered better predictors of ventricular arrhythmia than QTc. ${ }^{11}$ QTD was defined as the maximum QT interval minus the minimum QT interval. ${ }^{11-14}$ QTD reflects spatial differences in myocardial recovery time and has emerged as a noninvasive measurement for quantifying the degree of myocardial repolarization inhomogeneity. ${ }^{15,16}$ The use of QTD, which is the dispersion of the QT interval on a 12-lead ECG, as a surface ECG marker of vulnerability to ventricular arrhythmias and a potential predictor of cardiovascular mortality has been advocated. ${ }^{17}$ This variable comprises the difference between the shortest and longest QT interval on 12-lead surface ECG and has been recommended as a surrogate marker for the risk of arrhythmia in addition to the QT interval. ${ }^{18,19}$ QTDR, which is defined as QTD divided by cycle length, has been shown to be more predictive than QTD for ventricular arrhythmias. ${ }^{12}$ Tp-e represents the dispersion transmural repolarization time. Several experimental and clinical studies have suggested the utility of Tp-e as a marker of risk in arrhythmia syndromes, including congenital long QT syndrome. ${ }^{13,20} \mathrm{Tp}$-e is considered more useful than QTc. The Tp-e/QT ratio is significantly greater in patients at risk for an arrhythmic event, such as those with long QT syndrome. ${ }^{14}$ The Tp-e/QT ratio can predict cardiac arrhythmias and includes the values of transmural dispersion (Tp-e) and spatial dispersion (QT interval) of ventricular repolarization. ${ }^{21}$ The Tp-e/QT ratio is a more accurate predictor of ventricular arrhythmias than the QT interval, QTc or Tp-e. ${ }^{22}$ Furthermore, Tp-e, the Tp-e/QT ratio and the Tp-e/ QTc ratio are significantly higher in patients with acute myocarditis, which is thought to be a major cause of sudden cardiac death, compared with healthy controls. ${ }^{23}$ Based on these findings, we compared the effects of antidepressants on QTc, QTD, Tp-e and the Tp-e/QT ratio in 378 patients with mood disorder and indicated that the use of tricyclic antidepressants, especially clomipramine, was associated with prolonged QTc and QTD. ${ }^{24}$ However, information on such parameters in patients with schizophrenia treated with antipsychotics are limited.

Therefore, we attempted to clarify the effectiveness of these ECG indexes as predictors of ventricular arrhythmia associated with antipsychotic use. We sought to reevaluate the risk of sudden cardiac death due to antipsychotic use, especially the effects of the use of individual antipsychotics, by measuring these improved evaluation methods, namely, QTD, QTDR, Tp-e, the Tp-e/QT ratio, and the Tp-e/QTc ratio, in naturalistic settings. This study is a retrospective study that did not include controls who were not prescribed antipsychotics. We hypothesized that the use of antipsychotics drugs could affect the increase in the values of QTD, QTDR, Tp-e, Tp-e/QT ratio and Tp-e/ QTc ratio, which were regarded as more accurate predictors of ventricular arrhythmia in a dose-dependent manner.

\section{Patients and Methods}

\section{Participants}

The following data from 457 Japanese patients with schizophrenia treated with antipsychotics at Dokkyo Medical University, Fudougaoka Hospital and Kanuma Hospital between 2008 and 2020 were reviewed: medical history, ECG, and laboratory data. Among the 457 patients, 21 with hypokalemia $(<3.5 \mathrm{mEq} / \mathrm{L}), 3$ patients receiving treatment for hyperthyroidism, 15 with atrial fibrillation, 2 with a history of cardiac infarction and 6 receiving treatment for heart failure were excluded because these factors are well-known risk factors for QTc prolongation. Data from the remaining 410 patients (198 males; age range, 20-81 years; mean age 48.7 \pm 15.3 years) were examined in this study. A Torsade de points episode was not observed, and no patients died in this study period. 
Psychiatric diagnoses of schizophrenia were made based on the Diagnostic and Statistical Manual of Mental Disorders, 4th Edition, Text Revision, or 5th Edition. All patients underwent ECG to evaluate their physical condition and pharmacotherapy status. Finally, 235 healthy volunteers who had undergone routine health checkups at Dokkyo Medical University were recruited for this investigation. These participants had no physical abnormalities or abnormal laboratory data.

All participants were measured using the same ECG device in the supine position, and each index was investigated with the same methods to guarantee the validity of the results.

A standard 12-lead ECG was recorded at a paper speed of $25 \mathrm{~mm} / \mathrm{s}$. The QT interval was measured manually according to a previous report. ${ }^{25}$ The end of the $\mathrm{T}$ wave was determined as the intersection between the tangent to the steepest downslope of the $\mathrm{T}$ wave and the isoelectric line. If the height or depth of the $\mathrm{T}$ wave was less than $1.5 \mathrm{~mm}$, that lead was excluded from the analysis. Figure 1 presents some parameter's scheme used in this study.

The QT interval was then corrected as a value that varies in relation to heart rate. Several methods for correcting QT interval according to heart rate have been proposed, each with distinctive characteristics. In the present study, we used
Hodges's formula (QTc=QT interval $+1.75($ heart rate-60) $)$ because it is considered more appropriate than Bazett's formula $\left(\mathrm{QTc}=\mathrm{QT}\right.$ interval/ $\left./ \mathrm{RR}^{1 / 2}\right)$ in cases with tachycardia or bradycardia. ${ }^{5,26,27}$ QTD was calculated as the difference between the maximum and the minimum QT interval on a 12-lead ECG. QTDR is regarded as QTD divided by the cycle length in milliseconds and is expressed as a percentage. QTDR was defined by the formula (QTD/RR interval $) \times 100 .^{12}$ Tp-e was measured as the time from the top of $T$ wave to the end of the $\mathrm{T}$ wave (Figure 1). Tp-e and QT intervals were measured in lead V5. If V5 was not suitable, leads V4 and V6 were used, in that order. ${ }^{28}$ The Tp-e/QT ratio and Tp-e/QTc (Hogdes's formula) ratio were calculated as the ratio of Tp-e that led to the corresponding QT interval or QTc. ${ }^{22,23}$ The Tpe/QT ratio was calculated using the formula (Tp-e interval/QT interval) $\times 100$, and $\mathrm{Tp}-\mathrm{e} / \mathrm{QTc}$ ratio was calculated using the formula (Tp-e interval/QTc) $\times 100$.

\section{Medications}

Information on drugs administered was obtained from medical records. Administered drugs were unchanged for more than 1 week. Doses of antipsychotics prescribed to participants were converted to CP-equivalent doses. ${ }^{29}$ Antiparkinsonian drugs and benzodiazepines prescribed to participants were

\section{QT interval}

The time from the start of the $Q$ wave to the end of the $T$ wave.

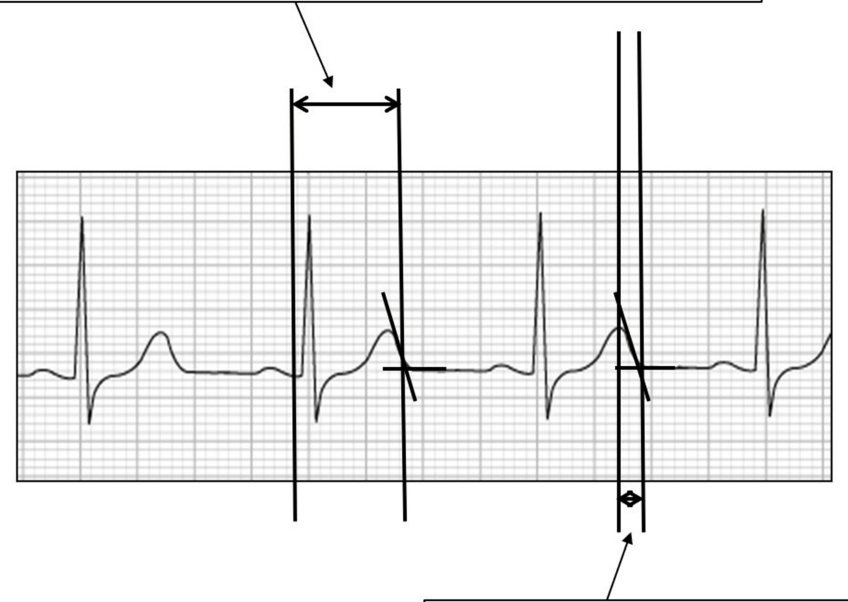

T wave peak-to-end (Tp-e)

The time from the top of $T$ wave to the end of the $T$ wave.

\section{QT dispersion (QTD)}

The difference between the maximum and the minimum QT interval on a 12-lead electrocardiography.

Figure I A scheme of explanation of QT interval, QT dispersion, T peak-to-end interval. 
converted to biperiden and diazepam equivalent doses, respectively. ${ }^{29}$ None of the subjects were coadministered nonpsychotropic drugs, such as antiarrhythmics, antibiotics, anticancer agents, antiemetics, and antihistamines that were associated with an increased risk of causing QT prolongation. ${ }^{30}$ A total of 27 patients were on antipsychotic monotherapy. A total of 363 patients prescribed antipsychotics along with one of the other psychotropic drugs, such as antiparkinsonian drugs, mood stabilizers, and benzodiazepines. A total of 112 patients were prescribed doses equivalent to greater than $1000 \mathrm{mg} \mathrm{CP}$. Table 1 presents the distribution of medications and dosages that were administered.

\section{Ethics}

This protocol for this study was approved by the ethics committees of Dokkyo Medical University (approval number:
$\mathrm{R}-27-3 \mathrm{~J})$. This study was conducted in accordance with the Declaration of Helsinki. All clinical records used in this study were anonymized by removing any identifying information (such as name and registration number). Furthermore, the clinical data were collated and managed by one person. The person who performed statistical analysis could not access the original data of each participant. Healthy controls underwent the examinations voluntarily. No children (under 20 years of age) were included in this study. Our study was approved, and written informed consent was not required from participants because this study was retrospective.

\section{Statistical Analysis}

For the first analysis, linear regression analysis was applied to examine risk factors for QTc lengthening, QTD, QTDR, Tp-e lengthening, Tp-e/QT ratio, and Tp-e/

Table I Demographic Data of Patients Diagnosed with Schizophrenia and Distribution of Medication and Dosage

\begin{tabular}{|c|c|c|c|c|c|c|}
\hline & \multicolumn{2}{|l|}{ Male } & \multicolumn{2}{|l|}{ Female } & \multicolumn{2}{|l|}{ Total } \\
\hline No. of patients & \multicolumn{2}{|l|}{198} & \multicolumn{2}{|l|}{212} & \multicolumn{2}{|l|}{410} \\
\hline Mean age (SD) & \multicolumn{2}{|l|}{$49.3(15.1)$} & \multicolumn{2}{|l|}{$48.2(15.6)$} & \multicolumn{2}{|l|}{$48.7(15.3)$} \\
\hline $\begin{array}{l}\text { Administered } \\
\text { Drugs }\end{array}$ & $\begin{array}{l}\text { No. of } \\
\text { Prescription (\%) }\end{array}$ & $\begin{array}{l}\text { Mean Dose } \\
\text { (SD) } \\
\text { mg }\end{array}$ & $\begin{array}{l}\text { No. of } \\
\text { Prescription (\%) }\end{array}$ & $\begin{array}{l}\text { Mean Dose } \\
\text { (SD) } \\
\text { mg }\end{array}$ & $\begin{array}{l}\text { No. of } \\
\text { Prescription (\%) }\end{array}$ & $\begin{array}{l}\text { Mean Dose } \\
\text { (SD) } \\
\text { mg }\end{array}$ \\
\hline $\begin{array}{l}\text { Antipsychotics } \\
\text { Chlorpromazine } \\
\text { Levomepromazine } \\
\text { Zotepine } \\
\text { Haloperidol } \\
\text { Sulpiride } \\
\text { Risperidone } \\
\text { Paliperidone } \\
\text { Perospirone } \\
\text { Quetiapine } \\
\text { Olanzapine } \\
\text { Aripiprazole } \\
\text { Blonanserin } \\
\text { Asenapine } \\
\text { Brexpiprazole } \\
\text { Clozapine }\end{array}$ & $\begin{array}{l}198(100.0) \\
50(25.3) \\
59(29.8) \\
18(9.09) \\
21(10.6) \\
2(1.01) \\
79(39.9) \\
21(10.6) \\
7(3.53) \\
32(16.2) \\
47(23.7) \\
42(21.2) \\
14(7.07) \\
2(1.01) \\
5(2.53) \\
3(1.52)\end{array}$ & $\begin{array}{l}792.9(517.0) \\
60.9(64.7) \\
72.5(71.7) \\
202.8(136.6) \\
9.29(7.06) \\
125.0(35.4) \\
5.92(2.93) \\
9.57(2.94) \\
27.4(19.1) \\
300.8(241.5) \\
15.5(6.08) \\
19.0(7.76) \\
15.7(5.54) \\
20.0(0) \\
2.0(0) \\
183.3(101.0)\end{array}$ & $\begin{array}{l}212(100.0) \\
31(14.6) \\
38(17.9) \\
19(8.96) \\
23(10.8) \\
2(0.94) \\
71(33.5) \\
17(8.02) \\
6(2.83) \\
19(8.96) \\
68(32.1) \\
43(20.3) \\
39(18.4) \\
14(6.60) \\
11(5.19) \\
1(0.47)\end{array}$ & $\begin{array}{l}725.1(499.8) \\
69.3(150.4) \\
53.2(49.0) \\
130.3(90.0) \\
8,54(8.52) \\
350.0(353.6) \\
5.0(3.15) \\
8.29(3.44) \\
19.3(8.91) \\
264.5(210.2) \\
14.0(5.83) \\
15.2(7.59) \\
16.8(7.06) \\
16.4(6.02) \\
2(0) \\
200\end{array}$ & $\begin{array}{l}410(100.0) \\
81(19.8) \\
97(23.7) \\
37(9.02) \\
44(10.7) \\
4(0.98) \\
150(36.6) \\
38(9.27) \\
13(3.17) \\
51(12.4) \\
115(28.0) \\
85(20.7) \\
53(12.9) \\
16(3.90) \\
16(3.90) \\
4(0.98)\end{array}$ & $\begin{array}{l}757.8(508.7) \\
64.2(105.2) \\
65.0(64.2) \\
165.5(119.2) \\
8.90(7.78) \\
237.5(242.8) \\
5.49(3.06) \\
9.00(3.20) \\
23.7(15.3) \\
287.3(228.9) \\
14.6(5.95) \\
17.1(7.87) \\
16.5(6.66) \\
16.9(5.74) \\
2.00(0.00) \\
187.5(82.9)\end{array}$ \\
\hline $\begin{array}{l}\text { Mood stabilizer } \\
\text { Lithium } \\
\text { Carbamazepine } \\
\text { Sodium valproate }\end{array}$ & $\begin{array}{l}32(16.1) \\
15(7.58) \\
49(24.7)\end{array}$ & $\begin{array}{l}634.4(213.4) \\
346.7(206.6) \\
613.3(389.8)\end{array}$ & $\begin{array}{l}22(10.4) \\
5(2.37) \\
32(15.2)\end{array}$ & $\begin{array}{l}563.6(217.2) \\
480.0(420.7) \\
509.4(238.8)\end{array}$ & $\begin{array}{l}54(13.2) \\
20(4.89) \\
81(19.8)\end{array}$ & $\begin{array}{l}605.6(215.8) \\
380.0(268.7) \\
572.2(340.4)\end{array}$ \\
\hline Benzodiazepines & I58 (79.8) & $19.3(14.5)$ & $167(78.8)$ & $16.6(14.0)$ & $325(79.3)$ & $17.9(14.3)$ \\
\hline Antiparkinsonian & II4 (57.6) & $2.85(1.66)$ & I0I (47.6) & $2.69(1.80)$ & $215(52.4)$ & 2.77 (I.72) \\
\hline
\end{tabular}

Notes: The antipsychotic dose was converted to chlorpromazine equivalents. The benzodiazepine dose was converted to diazepam equivalents. Antiparkinsonian dose was converted to biperiden equivalents.

Abbreviations: SD, standard deviation; No, number. 
QTc ratio. Age, sex, antipsychotic dose (chlorpromazine equivalent), antiparkinsonian drug dose (biperiden equivalent), benzodiazepine dose (diazepam equivalent) and mood stabilizer (lithium, sodium valproate, and carbamazepine) doses were entered as independent variables in the linear regression model. In the second analysis, age, sex, and individual antipsychotic doses were entered as independent variables in the linear regression model but antiparkinsonian drug, benzodiazepine or mood stabilizer doses were not entered as independent variables in the analysis. In the third analysis, QTc, QTD, QTDR, Tp-e, $\mathrm{Tp}$-e/QT ratio, and Tp-e/QTc ratio were compared between 235 patients prescribed antipsychotics and 235 healthy controls (142 males, mean age 56.1 \pm 10.8 ) adjusted for age and sex with a propensity score by Mann-Whitney U-test. All statistical analyses were performed using SPSS version 26.0 (IBM Japan, Tokyo, Japan). All reported $\mathrm{p}$ values are two-tailed, and statistical significance was set at $\mathrm{p}<0.05, \mathrm{p}<0.01$, and $\mathrm{p}<0.001$.

\section{Results QTc (Hodges's Formula)}

In the first analysis, age [partial regression coefficient (PRC) $=0.45,95 \%$ confidence interval $(\mathrm{CI})=0.27-0.63, \mathrm{p}<0.001]$, $\operatorname{sex}(\mathrm{PRC}=8.24,95 \% \mathrm{CI}=2.67-13.8, \mathrm{p}=0.038)$ and antipsychotics (chlorpromazine equivalents) ( $\mathrm{PRC}=1.00,95 \%$ $\mathrm{CI}=0.41-1.58, \mathrm{p}<0.001)$ were positively correlated with QTc (Hodges's formula) (Table 2). In the second analysis, age $\quad(\mathrm{PRC}=0.41, \quad 95 \% \quad \mathrm{CI}=0.23-0.59, \quad \mathrm{p}<0.001), \quad$ sex ( $\mathrm{PRC}=8.72,95 \% \mathrm{CI}=3.10-14.3, \mathrm{p}=0.0024)$, levomepromazine $(\mathrm{PRC}=13.2,95 \% \mathrm{CI}=6.54-19.8, \mathrm{p}<0.001)$, and brexpiprazole (PRC $=10.1,95 \% \mathrm{CI}=1.87-18.3, \mathrm{p}=0.016)$ were correlated with QTc (Hodges's formula) (Table 3).

Table 2 QTc (Hodges' Formula), QTD, QTDR, Tp-e, Tp-e/QT Ratio and Tp-e/QTc Ratio Effect of Distinct Neurotropic Drugs

\begin{tabular}{|c|c|c|c|c|c|c|}
\hline & $\begin{array}{l}\text { QTc (ms) } \\
\text { (Hodges's } \\
\text { Formula) }\end{array}$ & QTD (ms) & QTDR & Tp-e (ms) & Tp-e/QT Ratio & Tp-e/QTc Ratio \\
\hline & $\begin{array}{l}\text { Forced entry } \\
\text { PRC (95\% CI) }\end{array}$ & $\begin{array}{l}\text { Forced entry } \\
\text { PRC (95\% CI) }\end{array}$ & $\begin{array}{l}\text { Forced entry } \\
\text { PRC ( } 95 \% \mathrm{Cl})\end{array}$ & $\begin{array}{l}\text { Forced entry } \\
\text { PRC }(95 \% \mathrm{CI})\end{array}$ & $\begin{array}{l}\text { Forced entry } \\
\text { PRC (95\% Cl) }\end{array}$ & $\begin{array}{l}\text { Forced entry } \\
\text { PRC }(95 \% \mathrm{CI})\end{array}$ \\
\hline Age & $\begin{array}{l}0.45(0.27- \\
0.63)^{* * *}\end{array}$ & $\begin{array}{l}0.29(0.16- \\
0.43)^{* * *}\end{array}$ & $\begin{array}{l}0.023(0.0047- \\
0.042)^{*}\end{array}$ & $\begin{array}{l}0.25(0.12- \\
0.38)^{* * * *}\end{array}$ & $\begin{array}{l}0.016(-0.012- \\
0.043)\end{array}$ & $\begin{array}{l}0.032(0.0050- \\
0.058)^{*}\end{array}$ \\
\hline $\begin{array}{l}\text { Sex (risk in } \\
\text { women) }\end{array}$ & $\begin{array}{l}8.24(2.67- \\
13.8)^{*}\end{array}$ & $\begin{array}{l}2.24(-2.06- \\
6.55)\end{array}$ & $\begin{array}{l}0.57(-0.055- \\
\mathrm{I} .14)\end{array}$ & $\begin{array}{l}-3.92(-8.01- \\
0.17)\end{array}$ & $\begin{array}{l}-1.26(-2.11- \\
-0.04 I)^{* *}\end{array}$ & $\begin{array}{l}-1.41(-2.24- \\
-0.59)^{* * *}\end{array}$ \\
\hline $\begin{array}{l}\text { CP equivalent } \\
(100 \mathrm{mg})\end{array}$ & $\begin{array}{l}1.00(0.41- \\
1.58)^{* * *}\end{array}$ & $\begin{array}{l}0.46(0.010- \\
0.91)^{*}\end{array}$ & $\begin{array}{l}0.11(0.051- \\
0.17)^{* * *}\end{array}$ & $\begin{array}{l}0.64(0.21-1.07) \\
* *\end{array}$ & $\begin{array}{l}0.15(0.065- \\
0.24)^{* * *}\end{array}$ & $\begin{array}{l}0.092(0.0059- \\
0.18)^{*}\end{array}$ \\
\hline $\begin{array}{l}\text { Biperiden } \\
\text { equivalent } \\
(2 \mathrm{mg})\end{array}$ & $\begin{array}{l}-3.00(-6.21- \\
0.21)\end{array}$ & $\begin{array}{l}-2.08(-4.56- \\
0.40)\end{array}$ & $\begin{array}{l}-0.10(-0.43- \\
-0.23)\end{array}$ & $\begin{array}{l}-3.15(-5.51- \\
-0.80)^{* *}\end{array}$ & $\begin{array}{l}-0.51(-1.00- \\
-0.018)^{*}\end{array}$ & $\begin{array}{l}-0.57(-1.04- \\
-0.092)^{*}\end{array}$ \\
\hline $\begin{array}{l}\text { Lithium } \\
\text { (100 mg) }\end{array}$ & $\begin{array}{l}0.67(-0.64- \\
2.28)\end{array}$ & $\begin{array}{l}1.20(0.19- \\
2.22)^{*}\end{array}$ & $\begin{array}{l}0.086(-0.049- \\
0.22)\end{array}$ & $\begin{array}{l}1.52(0.56-2.49) \\
* *\end{array}$ & $\begin{array}{l}0.25(0.045- \\
0.45)^{*}\end{array}$ & $\begin{array}{l}0.32(0.13-0.5 I) \\
* *\end{array}$ \\
\hline $\begin{array}{l}\text { Sodium } \\
\text { valproate } \\
\text { (100 mg) }\end{array}$ & $0.14(-0.88-1.16)$ & $\begin{array}{l}0.50(-0.29- \\
1.29)\end{array}$ & $\begin{array}{l}0.074(-0.031- \\
0.18)\end{array}$ & $\begin{array}{l}-0.24(-0.99- \\
0.51)\end{array}$ & $\begin{array}{l}-0.07 \mid(-0.23- \\
0.086)\end{array}$ & $\begin{array}{l}-0.062(-0.21- \\
0.089)\end{array}$ \\
\hline $\begin{array}{l}\text { Carbamazepine } \\
\text { (100 mg) }\end{array}$ & $\begin{array}{l}-0.55(-3.34- \\
2.28)\end{array}$ & $\begin{array}{l}1.89(-0.30- \\
4.07)\end{array}$ & $\begin{array}{l}0.35(0.056- \\
0.64)^{*}\end{array}$ & $\begin{array}{l}-1.22(-3.23- \\
0.85)\end{array}$ & $\begin{array}{l}-0.30(-0.73- \\
0.13)\end{array}$ & $\begin{array}{l}-0.26(-0.68- \\
0.15)\end{array}$ \\
\hline $\begin{array}{l}\text { Diazepam } \\
\text { equivalent } \\
(5 \mathrm{mg})\end{array}$ & $\begin{array}{l}-0.15(-1.14- \\
0.84)\end{array}$ & $\begin{array}{l}0.56(-0.21- \\
\mathrm{I} .33)\end{array}$ & $\begin{array}{l}0.071(-0.032- \\
0.17)\end{array}$ & $\begin{array}{l}-00.20(-0.75- \\
0.7 I)\end{array}$ & $\begin{array}{l}0.026(-0.13- \\
0.18)\end{array}$ & $\begin{array}{l}-0.0021(-0.15- \\
0.15)\end{array}$ \\
\hline
\end{tabular}

Notes: Age, sex (risk in women) and antipsychotics were positively correlated with QTc. Age, antipsychotics and lithium were positively correlated with QTD. Age, antipsychotics and carbamazepine were positively correlated with QTDR. Age, antipsychotics and lithium were positively correlated with Tp-e. Antipsychotics and lithium were positively correlated with the Tp-e/QT ratio. Age, antipsychotics and lithium were positively correlated with the Tp-e/QTc ratio. Sex was negatively correlated with the Tp-e/QT ratio and Tp-e/QTc ratio. ${ }^{*} \mathrm{p}<0.05,{ }^{* *} \mathrm{p}<0.01$, $* * * \mathrm{p}<0.001$.

Abbreviations: QTc, corrected QT interval; QTD, QT dispersion; QTDR, QTD rate; TP-e, T peak-to-end interval; PRC, partial regression coefficient; CI, confidence interval; $\mathrm{CP}$, chlorpromazine. 
Table 3 QTc (Hodges' Formula), QTD, QTDR, Tp-e, Tp-e/QT Ratio, and Tp-e/QTc Ratio Effects of Individual Antipsychotic Drugs

\begin{tabular}{|c|c|c|c|c|c|c|}
\hline & $\begin{array}{l}\text { QTc (ms) } \\
\text { (Hodges's } \\
\text { Formula) }\end{array}$ & QTD (ms) & QTDR & Tp-e (ms) & Tp-e/QT Rate & Tp-e/QTc Rate \\
\hline & $\begin{array}{l}\text { Forced entry } \\
\text { PRC (95\% CI) }\end{array}$ & $\begin{array}{l}\text { Forced entry } \\
\text { PRC (95\% Cl) }\end{array}$ & $\begin{array}{l}\text { Forced entry } \\
\text { PRC }(95 \% \mathrm{CI})\end{array}$ & $\begin{array}{l}\text { Forced entry } \\
\text { PRC }(95 \% \mathrm{CI})\end{array}$ & $\begin{array}{l}\text { Forced entry } \\
\text { PRC }(95 \% \mathrm{CI})\end{array}$ & $\begin{array}{l}\text { Forced entry } \\
\text { PRC }(95 \% \mathrm{Cl})\end{array}$ \\
\hline Age & $\begin{array}{l}0.4 I(0.23-0.59) \\
* * *\end{array}$ & $\begin{array}{l}0.28(0.14-0.43) \\
* * *\end{array}$ & $\begin{array}{l}0.021(0.0023- \\
0.040)^{*}\end{array}$ & $\begin{array}{l}0.24(0.10-0.37) \\
* * *\end{array}$ & $\begin{array}{l}0.014(-0.014- \\
0.042)\end{array}$ & $\begin{array}{l}0.031(0.0037- \\
0.58)^{*}\end{array}$ \\
\hline $\begin{array}{l}\text { Sex (risk in } \\
\text { women) }\end{array}$ & $8.72(3.10-14.3)^{* *}$ & $\begin{array}{l}1.49(-3.01- \\
6.00)\end{array}$ & $\begin{array}{l}0.53(-0.060- \\
I .12)\end{array}$ & $\begin{array}{l}-4.41(-8.57- \\
-0.26)^{*}\end{array}$ & $\begin{array}{l}-1.32(-2.20- \\
-0.44)^{* *}\end{array}$ & $\begin{array}{l}-1.56(-2.40- \\
-0.7 I)^{* * *}\end{array}$ \\
\hline $\begin{array}{l}\text { Chlorpromazine } \\
(100 \mathrm{mg})\end{array}$ & $\begin{array}{l}-0.53(-5.70- \\
4.64)\end{array}$ & $\begin{array}{l}1.54(-2.60- \\
5.68)\end{array}$ & $\begin{array}{l}0.46(-0.087- \\
1.00)\end{array}$ & $\begin{array}{l}-0.095(-3.92- \\
3.73)\end{array}$ & $\begin{array}{l}0.31(-0.49- \\
\text { I.I2) }\end{array}$ & $\begin{array}{l}-0.0057(-0.78- \\
0.77)\end{array}$ \\
\hline $\begin{array}{l}\text { Levomepromazine } \\
\text { (100 mg) }\end{array}$ & $\begin{array}{l}\text { I3.2 (6.54 - 19.8) } \\
* * *\end{array}$ & $\begin{array}{l}7.49(2.17-12.8) \\
* *\end{array}$ & $\begin{array}{l}1.12(0.42-1.81) \\
* *\end{array}$ & $\begin{array}{l}5.48(0.58-10.4) \\
*\end{array}$ & $\begin{array}{l}0.47(-0.56- \\
\mathrm{I} .5 \mathrm{I})\end{array}$ & $\begin{array}{l}0.39(-0.60- \\
\text { I.39) }\end{array}$ \\
\hline Zotepine (66 mg) & $2.72(-0.38-5.83)$ & $\begin{array}{l}0.48(-2.01- \\
2.97)\end{array}$ & $\begin{array}{l}0.31(-0.014- \\
0.64)\end{array}$ & $\begin{array}{l}-0.74(-3.05- \\
\text { I.55) }\end{array}$ & $\begin{array}{l}-0.11(-0.60- \\
0.37)\end{array}$ & $\begin{array}{l}-0.32(-0.79- \\
0.14)\end{array}$ \\
\hline Haloperidol (2 mg) & $0.31(-1.19-1.80)$ & $\begin{array}{l}0.90(-0.30- \\
2.10)\end{array}$ & $\begin{array}{l}0.11(-0.045- \\
0.27)\end{array}$ & $\begin{array}{l}0.63(-0.47- \\
1.74)\end{array}$ & $\begin{array}{l}0.14(-0.094- \\
0.37)\end{array}$ & $\begin{array}{l}0.13(-0.097- \\
0.35)\end{array}$ \\
\hline Sulpiride $(200 \mathrm{mg})$ & $13.6(-3.69-31.0)$ & $\begin{array}{l}-5.13(-19.0- \\
8.75)\end{array}$ & $\begin{array}{l}-0.15(-1.97- \\
1.67)\end{array}$ & $\begin{array}{l}3.59(-9.23- \\
16.4)\end{array}$ & $\begin{array}{l}0.77(-1.94- \\
3.47)\end{array}$ & $\begin{array}{l}0.017(-9.68- \\
2.61)\end{array}$ \\
\hline Risperidone (I mg) & $0.55(-0.38-1.48)$ & $\begin{array}{l}0.19(-0.55- \\
0.94)\end{array}$ & $\begin{array}{l}0.081(-0.016- \\
0.18)\end{array}$ & $\begin{array}{l}0.48(-0.21- \\
\mathrm{I} .17)\end{array}$ & $\begin{array}{l}0.14(-0.064- \\
0.28)\end{array}$ & $\begin{array}{l}0.093(-0.47- \\
0.23)\end{array}$ \\
\hline $\begin{array}{l}\text { Paliperidone } \\
(1.5 \mathrm{mg})\end{array}$ & $0.28(-1.22-1.78)$ & $\begin{array}{l}0.18(-1.01- \\
1.39)\end{array}$ & $\begin{array}{l}0.052(-0.11- \\
0.21)\end{array}$ & $\begin{array}{l}0.86(-0.24- \\
1.97)\end{array}$ & $\begin{array}{l}0.21(-0.019- \\
0.45)\end{array}$ & $\begin{array}{l}0.196(-0.028- \\
0.43)\end{array}$ \\
\hline Perospirone (8 mg) & $\begin{array}{l}-0.88(-5.29- \\
3.53)\end{array}$ & $\begin{array}{l}-2.25(-5.77- \\
\text { I.29) }\end{array}$ & $\begin{array}{l}-0.21(-0.67- \\
0.26)\end{array}$ & $\begin{array}{l}-2.69(-5.95- \\
0.57)\end{array}$ & $\begin{array}{l}-0.58(-1.27- \\
0.11)\end{array}$ & $\begin{array}{l}-0.60(-1.26- \\
-0.06 \mathrm{I})\end{array}$ \\
\hline $\begin{array}{l}\text { Quetiapine } \\
(66 \mathrm{mg})\end{array}$ & $\begin{array}{l}-0.47(-1.91- \\
0.97)\end{array}$ & $\begin{array}{l}0.61(-0.54- \\
I .77)\end{array}$ & $\begin{array}{l}0.18(0.033- \\
0.34)^{*}\end{array}$ & $\begin{array}{l}-0.81(-1.88- \\
0.25)\end{array}$ & $\begin{array}{l}-0.064(-0.29- \\
0.16)\end{array}$ & $\begin{array}{l}-0.17(-0.38- \\
0.050)\end{array}$ \\
\hline $\begin{array}{l}\text { Olanzapine } \\
(2.5 \mathrm{mg})\end{array}$ & $\begin{array}{l}0.92(-0.076- \\
1.91)\end{array}$ & $\begin{array}{l}0.49(-0.31- \\
\text { I.29) }\end{array}$ & $\begin{array}{l}0.79(-0.026- \\
0.18)\end{array}$ & $\begin{array}{l}1.22(0.49-1.96) \\
* *\end{array}$ & $\begin{array}{l}0.27(0.12-0.43) \\
* * *\end{array}$ & $\begin{array}{l}0.22(0.076- \\
0.37)^{* *}\end{array}$ \\
\hline $\begin{array}{l}\text { Aripiprazole } \\
(4 \mathrm{mg})\end{array}$ & $\begin{array}{l}-0.15(-1.63- \\
1.33)\end{array}$ & $\begin{array}{l}0.52(-0.66- \\
1.70)\end{array}$ & $\begin{array}{l}0.098(-0.057- \\
0.25)\end{array}$ & $\begin{array}{l}-0.055(-1.10- \\
1.08)\end{array}$ & $\begin{array}{l}0.083(-0.15- \\
0.31)\end{array}$ & $\begin{array}{l}0.024(-0.20- \\
0.25)\end{array}$ \\
\hline Blonanserin (4 mg) & $0.18(-1.71-2.80)$ & $\begin{array}{l}0.73(-0.78- \\
2.24)\end{array}$ & $\begin{array}{l}0.17(-0.032- \\
0.36)\end{array}$ & $\begin{array}{l}0.20(-1.20- \\
1.60)\end{array}$ & $\begin{array}{l}0.077(-0.22- \\
0.37)\end{array}$ & $\begin{array}{l}0.027(-0.26- \\
0.31)\end{array}$ \\
\hline Asenapine $(2.5 \mathrm{mg})$ & $0.48(-1.84-2.07)$ & $\begin{array}{l}1.19(-0.67- \\
3.04)\end{array}$ & $\begin{array}{l}0.28(0.032- \\
0.52)^{*}\end{array}$ & $\begin{array}{l}-0.57(-2.29- \\
\text { I.I4) }\end{array}$ & $\begin{array}{l}-0.096(-0.46- \\
0.27)\end{array}$ & $\begin{array}{l}-0.16(-0.51- \\
0.19)\end{array}$ \\
\hline $\begin{array}{l}\text { Brexpiprazole } \\
\text { (I mg) }\end{array}$ & $10.1(1.87-18.3)^{*}$ & $\begin{array}{l}0.42(-6.19- \\
7.03)\end{array}$ & $\begin{array}{l}-0.44(-1.31- \\
0.43)\end{array}$ & $\begin{array}{l}\text { II.8 (5.67 - I7.9) } \\
\text { *** }\end{array}$ & $\begin{array}{l}1.90(0.61-3.19) \\
* *\end{array}$ & $\begin{array}{l}2.15(0.91-3.39) \\
* * *\end{array}$ \\
\hline Clozapine (50 mg) & $3.26(-3.59-10.1)$ & $\begin{array}{l}2.31(-3.18- \\
7.80)\end{array}$ & $\begin{array}{l}0.86(0.14-1.58) \\
*\end{array}$ & $\begin{array}{l}-0.98(-6.04- \\
4.09)\end{array}$ & $\begin{array}{l}0.19(-0.88- \\
1.25)\end{array}$ & $\begin{array}{l}-0.41(-1.44- \\
0.62)\end{array}$ \\
\hline
\end{tabular}

Notes: Age, sex (risk in women), levomepromazine and brexpiprazole were positively correlated with QTc. Age and levomepromazine were positively correlated with QTD. Age, levomepromazine, quetiapine, asenapine and clozapine were positively correlated with QTDR. Age, levomepromazine, olanzapine and brexpiprazole were positively correlated with Tp-e. Olanzapine and brexpiprazole were positively correlated with the Tp-r/QT ratio. Age, olanzapine and brexpiprazole were positively correlated with the Tp-e/QTc ratio. Sex was negatively correlated with Tp-e, Tp-e/QT and Tp-e/QTc. *p<0.05, **p<0.0I, *** ${ }^{*}<0.001$.

Abbreviations: QTc, corrected QT interval; QTD, QT dispersion; QTDR, QTD rate; Tp-e, T peak-to-end interval; PRC, partial regression coefficient; Cl, confidence interval. 


\section{QTD}

In the first analysis, age ( $\mathrm{PRC}=0.29,95 \% \mathrm{CI}=0.16-0.43$, $\mathrm{p}<0.001$ ), antipsychotics ( $\mathrm{PRC}=0.46,95 \% \mathrm{CI}=0.010-0.91$, $\mathrm{p}=0.045)$ and lithium ( $\mathrm{PRC}=1.20,95 \% \quad \mathrm{CI}=0.19-2.22$, $\mathrm{p}=0.021$ ) were positively correlated with QTD (Table 2). In the second analysis, age $(\mathrm{PRC}=0.28,95 \% \mathrm{CI}=0.14-0.43$, $\mathrm{p}<0.001)$ and levomepromazine $\quad(\mathrm{PRC}=7.49, \quad 95 \%$ $\mathrm{CI}=2.17-12.8, \mathrm{p}=0.0058$ ) were correlated with QTD (Table 3).

\section{QTDR}

In the first analysis, age ( $\mathrm{PRC}=0.023,95 \% \mathrm{CI} 95 \%$ $\mathrm{CI}=0.0047-0.042, \mathrm{p}=0.014)$, antipsychotics $\quad(\mathrm{PRC}=0.11$, 95\% $\quad \mathrm{CI}=0.051-0.17, \quad \mathrm{p}<0.001) \quad$ and carbamazepine ( $\mathrm{PRC}=0.35$ 95\% $\mathrm{CI}=0.056-0.64, \mathrm{p}=0.019$ ) were positively correlated with QTDR (Table 2). In the second analysis, age ( $\mathrm{PRC}=0.021 .95 \% \mathrm{CI}=0.0023-0.040, \mathrm{p}=0.028)$, levomepromazine $(\mathrm{PRC}=1.12,95 \% \mathrm{CI}=0.42-1.81, \mathrm{p}=0.0017)$, quetiapine $(\mathrm{PRC}=0.18,95 \% \mathrm{CI}=0.033-0.34, \mathrm{p}=0.017)$, asenapine $(\mathrm{PRC}=0.28,95 \% \quad \mathrm{CI}=0.032-0.52, \mathrm{p}=0.027)$, and clozapine $(\mathrm{PRC}=0.86,95 \% \mathrm{CI}=0.14-1.58, \mathrm{p}=0.020)$ were correlated with QTDR (Table 3).

\section{Tp-e}

In the first analysis, age ( $\mathrm{PRC}=0.25,95 \% \mathrm{CI}=0.12-0.38$, $\mathrm{p}<0.001$ ), antipsychotics ( $\mathrm{PRC}=0.64,95 \% \mathrm{CI} 95 \% \mathrm{CI}=0.21$ $-1.07, \mathrm{p}=0.0036)$, and lithium $(\mathrm{PRC}=1.52,95 \% \mathrm{CI}=0.56$ $-2.49, \mathrm{p}=0.0020$ ) were positively correlated with $\mathrm{Tp}$-e. On the other hand, antiparkinsonian drugs (biperiden equivalent) ( $\mathrm{PRC}=-3.15,95 \% \mathrm{CI} 95 \% \mathrm{CI}=-5.51--0.80, \mathrm{p}=0.0088$ ) were negatively correlated with $\mathrm{Tp}$-e (Table 2). In the second analysis, age ( $\mathrm{PRC}=0.24,95 \% \mathrm{CI}=0.10-0.37, \mathrm{p}<0.001)$, levomepromazine $\quad(\mathrm{PRC}=5.48, \quad 95 \% \quad \mathrm{CI}=0.58-10.4, \quad \mathrm{p}=0.029)$, olanzapine $(\mathrm{PRC}=1.22,95 \% \mathrm{CI}=0.49-1.96, \mathrm{p}=0.0012)$ and brexpiprazole $(\mathrm{PRC}=11.8,95 \% \mathrm{CI}=5.67-17.9, \quad \mathrm{p}<0.001)$ were correlated with Tp-e. On the other hand, sex (PRC= $-4.41,95 \% \mathrm{CI} 95 \% \mathrm{CI}=-8.57--0.26, \mathrm{p}=0.038)$ was negatively correlated with Tp-e. The prolonged risk of Tp-e was higher in men than in women (Table 3).

\section{Tp-e/QT Ratio}

In the first analysis, antipsychotics ( $\mathrm{PRC}=0.15,95 \% \mathrm{CI} 95 \%$ $\mathrm{CI}=0.065-0.24, \mathrm{p}<0.001)$ and lithium ( $\mathrm{PRC}=0.25,95 \%$ $\mathrm{CI} 95 \% \mathrm{CI}=0.045-0.45, \mathrm{p}=0.016)$ were positively correlated with the Tp-e/QT ratio. On the other hand, sex (PRC= $-1.26,95 \% \mathrm{CI} 95 \% \mathrm{CI}=-2.11-0.041, \mathrm{p}=0.0038)$ and antiparkinsonian drugs $(\mathrm{PRC}=-0.51,95 \% \mathrm{CI} 95 \% \mathrm{CI}=-1.00-0.018$, $\mathrm{p}=0.042$ ) were negatively correlated with the Tp-e/QT ratio
(Table 2). In the second analysis, olanzapine ( $\mathrm{PRC}=0.27,95 \%$ $\mathrm{CI} 95 \% \quad \mathrm{CI}=0.12-0.43, \quad \mathrm{p}<0.001) \quad$ and brexpiprazole ( $\mathrm{PRC}=1.90,95 \% \mathrm{CI} 95 \% \mathrm{CI}=0.61-3.19, \mathrm{p}=0.0039)$ were positively correlated with the Tp-e/QT ratio. On the other hand, $\operatorname{sex}(\mathrm{PRC}=-1.32,95 \% \mathrm{CI} 95 \% \mathrm{CI}=-2.20-0.44, \mathrm{p}=0.0033)$ was negatively correlated with the Tp-e/QT ratio (Table 3).

\section{Tp-e/QTc Ratio}

In the first analysis, age ( $\mathrm{PRC}=0.032,95 \% \mathrm{CI} 95 \% \mathrm{CI}=0.0050$ $-0.058, \mathrm{p}=0.020$ ), antipsychotics ( $\mathrm{PRC}=0.092,95 \%$ CI $95 \%$ $\mathrm{CI}=0.0059-0.18, \mathrm{p}=0.036)$ and lithium $(\mathrm{PRC}=0.32,95 \%$ $\mathrm{CI} 95 \% \mathrm{CI}=0.13-0.51, \mathrm{p}=0.0013)$ were positively correlated with the Tp-e/QTc ratio. On the other hand, sex (PRC= $-1.41,95 \% \mathrm{CI} 95 \% \mathrm{CI}=-2.24-0.59, \mathrm{p}<0.001)$ and antiparkinsonian drugs $(\mathrm{PRC}=-0.57,95 \% \mathrm{CI} 95 \% \mathrm{CI}=-1.04-0.092$, $\mathrm{p}=0.019$ ) were negatively correlated with the $\mathrm{Tp}-\mathrm{e} / \mathrm{QTc}$ ratio (Table 2). In the second analysis, age ( $\mathrm{PRC}=0.031,95 \% \mathrm{CI}$ $95 \% \mathrm{CI}=0.0037-0.58, \mathrm{p}=0.027$ ), olanzapine ( $\mathrm{PRC}=0.22,95 \%$ $\mathrm{CI} 95 \% \mathrm{CI}=0.076-0.37, \quad \mathrm{p}=0.0033)$ and brexpiprazole ( $\mathrm{PRC}=2.15,95 \% \mathrm{CI} 95 \% \mathrm{CI}=0.91-3.39, \mathrm{p}<0.001)$ were positively correlated with the Tp-e/QTc ratio. On the other hand, sex $(\mathrm{PRC}=-1.56,95 \% \mathrm{CI} 95 \% \mathrm{CI}=-2.40--0.71, \mathrm{p}<0.001)$ was negatively correlated with the Tp-e/QTc ratio (Table 3 ).

\section{Comparison Between Patients Prescribed Antipsychotics and Healthy Controls}

In the third analysis, significant differences in QTc $(\mathrm{p}<0.001)$, QTD $(\mathrm{p}<0.026)$, QTDR $(\mathrm{p}<0.001), \quad$ Tp-e $(\mathrm{p}<0.001), \mathrm{Tp}-\mathrm{e} / \mathrm{QT}$ ratio $(\mathrm{p}<0.001)$ and $\mathrm{Tp}-\mathrm{e} / \mathrm{QTc}$ ratio $(p<0.001)$ were found between patients and healthy controls (Table 4).

\section{Discussion}

The results of this study regarding medication were as follows. Positive correlations were noted between QTc and levomepromazine and brexpiprazole. Levomepromazine and lithium were positively correlated with QTD. Levomepromazine, quetiapine, asenapine, clozapine and carbamazepine were positively correlated with QTDR. Levomepromazine, olanzapine, brexpiprazole and lithium were positively correlated with Tp-e. Olanzapine, brexpiprazole and lithium were positively correlated with the Tp-e/QT ratio. Olanzapine, brexpiprazole and lithium were positively correlated with Tp-e/QTc ratio. In addition, significant differences in QTc, QTD, QTDR, Tp-e, Tp-e/QT ratio and Tp-e/ QTc ratio were found between the patients and healthy controls. Our findings suggest that proarrhythmic risk markers 
Table 4 Comparison of QTc, QT Dispersion (QTD), QTD Rate (QTDR), T Wave Peak-to-End Interval (Tp-e), Tp-e/QT and Tpe/QTc Between Patients Treated with Drugs and Healthy Controls Adjusted for Age and Sex with Propensity Score

\begin{tabular}{|l|l|l|l|}
\hline & $\begin{array}{l}\text { Schizophrenia } \\
\text { Patients }\end{array}$ & $\begin{array}{l}\text { Healthy } \\
\text { Controls }\end{array}$ & \multirow{2}{*}{ p-value } \\
\cline { 2 - 3 } & Mean (SD) & Mean (SD) & \\
\hline $\begin{array}{l}\text { QTc (Hodges's } \\
\text { formula) }\end{array}$ & $417.7(30.0)$ & $393.8(26.2)$ & $<0.00 I^{* *}$ \\
\hline QTD & $57.6(23.5)$ & $51.9(19.1)$ & $0.026^{*}$ \\
\hline QTDR & $6.93(3.14)$ & $5.69(2.30)$ & $<0.00 I^{* *}$ \\
\hline Tp-e & $98.4(24.1)$ & $89.1(49.3)$ & $<0.00 I^{* *}$ \\
\hline Tp-e/QT & $24.8(4.7 I)$ & $23.3(12.7)$ & $<0.00 I^{* *}$ \\
\hline $\begin{array}{l}\text { Tp-e/QTc (Hodges's } \\
\text { formula) }\end{array}$ & $23.5(4.60)$ & $22.7(12.5)$ & $<0.00 I^{* *}$ \\
\hline
\end{tabular}

Notes: Significant differences in QTc, QTD, QTDR, Tp-e, Tp-e/QT and Tp-e/QTc were identified between patients and healthy controls. ${ }^{p} p<0.05,{ }^{*} p<0.001$.

were the presence of schizophrenia and the use of antipsychotics. Given that the results regarding QTc, QTD, QTDR, $\mathrm{Tp}-\mathrm{e}, \mathrm{Tp}-\mathrm{e} / \mathrm{QT}$ ratio and Tp-e/QTc ratio were inconsistent in this study, QTc alone might not reflect the risk of sudden cardiac death. Actually, we confirmed that quetiapine, asenapine and clozapine were positively correlated with QTD and QTDR, and olanzapine was positively correlated with Tp-e, Tp-e/QT ratio and Tp-e/QTc ratio despite not being correlated with QTc in our research. Therefore, the predictive factor of ventricular arrhythmia on ECG should be evaluated by using various indexes, such as QTD, QTDR, Tp-e, Tp-e/ QT ratio and Tp-e/QTc ratio.

The present study identified antipsychotic use as a potential risk factor for prolonged increased QTc, QTD, QTDR, Tp-e, Tp-e/QT ratio and Tp-e/QTc ratio. Among the distinct antipsychotic drugs, levomepromazine influenced QTD, QTDR and Tp-e; quetiapine, asenapine and clozapine influenced QTDR; and olanzapine and brexpiprazole influenced Tp-e, Tp-e/QT ratio and Tp-e/Qc ratio in a dose-dependent manner. QTD indicates dispersion of surface depolarization, whereas Tp-e represents transmural repolarization. Therefore, QTD and Tp-e each might have different physiological implications. Levomepromazine, quetiapine, asenapine and clozapine could affect spatial dispersion evaluated by QTD or QTDR. On the other hand, olanzapine and brexpiprazole only affected transmural repolarization evaluated by Tp-e, $\mathrm{Tp}-\mathrm{e} / \mathrm{QT}$ ratio and $\mathrm{Tp}-\mathrm{e} / \mathrm{QTc}$ ratio. In particular, levomepromazine, quetiapine, olanzapine and clozapine influenced QTDR, which is a more predictive factor than QTc or QTD for ventricular arrhythmias, and olanzapine and brexpiprazole influenced $\mathrm{Tp}-\mathrm{e} / \mathrm{QT}$ ratio or $\mathrm{Tp}$-e/QTc ratio, which are more predictive factors than QTc or Tp-e. These drugs might be associated with a relatively higher risk for inducing ventricular arrhythmias compared with other antipsychotics. Thus, each antipsychotic agent could affect heart conduction through different pharmacological mechanisms. However, the findings related to asenapine, brexpipraze and clozapine should be carefully interpreted because there was a small number of patients administered these drugs. In fact, brexpiprazole is known to have no effect on QTc prolongation. ${ }^{31}$ Our previous study demonstrated that levomepromazine significantly lengthened QTc, whereas second-generation antipsychotics (risperidone, olanzapine and quetiapine) did not prolong QTc. ${ }^{7}$ Furthermore, it has been suggested that aripiprazole, brexpiprazole, and olanzapine do not increase QT interval despite the low quality of evidence, ${ }^{32}$ while the United States Food and Drug Administration (US FDA) warned that atypical antipsychotics (olanzapine, aripiprazole, risperidone, and quetiapine) increase the risk of sudden cardiac death in the elderly by $4.5 \% .{ }^{33}$ In addition, sudden cardiac death has been associated with exposure to both first- and second-generation antipsychotics. ${ }^{34}$ Therefore, it should be confirmed whether first- or second-generation antipsychotics can be risk factors for sudden cardiac death by using QTD, QTDR, Tp-e, the Tp-e/QT ratio and the $\mathrm{Tp}$-e/QTc ratio in addition to QTc prolongation.

In a previous study, the QTc interval was longer in women than in men, but the QTD was similar in men and women among healthy subjects, ${ }^{35}$ and the Tp-e interval did not differ according to sex. ${ }^{36}$ Our results were similar to those in a previous report on QTc and QTD; however, contrary to a previous report in a healthy person, $\mathrm{Tp}$-e was longer in men than in women in schizophrenia patients. Unlike healthy people, some factors could affect transmural repolarization in the patients of men with schizophrenia. Acciavatti et al reported the association of psychotropic drugs and ventricular repolarization with QT interval, Tp-e and QTD on ECG in various psychiatric disorders, such as schizophrenia, bipolar disorder, depressive disorder, personality disorder, substance-related disorder and intellectual disability. ${ }^{37} \mathrm{In}$ that report, methadone, clozapine and lithium use were associated with Tp-e, and piperazine phenothiazines increased QTD. Haloperidol; aliphatic phenothiazines, such as chlorpromazine and levomepromazine; and other second- 
generation antipsychotics except for clozapine were not associated with QTc, Tp-e or QTD. ${ }^{37}$ Our results were similar to those of a previous report on the association between lithium use and the prolongation of QTD and Tp-e. In contrast, the conclusion about the association between antipsychotic use and the various ECG indexes differed from that in Acciavatti's report. Our report showed that antipsychotics use prolonged QTc, QTD, QTDR, Tp-e, Tp-e/QT ratio and $\mathrm{Tp}-\mathrm{e} / \mathrm{QTc}$ ratio in a dose-dependent manner. Although further research is necessary, the heart conduction of Japanese patients with schizophrenia could be easily affected constitutionally. In addition, the fact that there are still many patients with schizophrenia who are administered polypharmacy rather than high-dose antipsychotic in Japan could be one reason for the prolongation of various indexes on ECG measured in this study. ${ }^{38}$

Lithium is a drug that prolongs QT interval. ${ }^{39}$ On the other hand, multiple case series exhibit no significant change in the QTc interval of patients on lithium therapy before and after treatment initiation with lithium levels in the therapeutic range $(0.6-1.2 \mathrm{mEq} / \mathrm{L}){ }^{6}{ }^{6}$ In our results, the concomitant use of lithium was positively associated with QTD, Tp-e and the Tp-e/QTc ratio but not with QTc or QTDR in patients with schizophrenia. Carbamazepine was associated with sudden unexpected death in epilepsy patients, but the mechanism was uncertain. ${ }^{40}$ Our results revealed a positive correlation between the concomitant use of carbamazepine and QTDR.

The strength of our present study is that it showed associations with various indexes beyond the QTc on the ECG in schizophrenia patients who used antipsychotics and other psychotropic drugs in a natural setting retrospectively. In addition, this was the first study to analyze an association between the dose of independent antipsychotics and the various ECG indexes, in particular QTDR and $\mathrm{Tp}-\mathrm{e} / \mathrm{QTc}$ ratio.

Based on the above finding, our research demonstrated that it is not possible to predict ventricular arrhythmias by only measuring the QTc. Thus, we think that it is more desirable to measure QTc together with other parameters, such as QTD, QTDR, Tp-e, Tp-e/QT ratio and Tp-e/QTc ratio, which are more predictive of ventricular arrhythmias than QTc in patients with schizophrenia administered antipsychotic drugs daily in our study.

\section{Limitations}

There are several limitations to this study. First, because we evaluated real-world patients retrospectively, we could not evaluate changes in QTc, QTD, QTDR, Tp-e, Tp-e/QT ratio, and $\mathrm{Tp}-\mathrm{e} / \mathrm{QTc}$ ratio before and after medication use in the same patients. Second, we did not adjust for the time of ECG measurement. Third, we compared QTc, QTD, QTDR, Tp-e, Tp-e/QT ratio, and Tp-e/QTc ratio between patients treated with drugs and healthy controls, but these were not comparisons between medication-naïve schizophrenia patients and healthy controls or schizophrenia patients treated with drugs.

\section{Conclusion}

Although this research has some limitations mentioned above, we indicated that antipsychotic drug use affected various indexes of ventricular arrhythmia on ECG in a dose-dependent manner. For the safe use of antipsychotic medication, it is necessary for the proarrhythmic effect of antipsychotic drugs to be evaluated using various indexes, such as QTD, QTDR, Tp-e, Tp-e/QT ratio, and Tp-e/QTc ratio. To avoid the risk of ventricular arrhythmia, we have to assess the risk of the prolongation of these indexes according to the characteristics of the drug used. To reach a firm conclusion on this matter, larger-scale cohort studies are required. A comparison between medicationnaïve schizophrenia patients and healthy controls or schizophrenia patients treated with antipsychotics is required.

\section{Acknowledgments}

This study was supported by a Grant-in-Aid for Scientific Research from the Japan Society for the Promotion of Research (JSPS KAKENHI) (Grant Number JP15K19745).

This funding source had no role in the design of this study and did not have any role in its execution, analyses, interpretation of the data, or decision to submit results.

\section{Author Contributions}

All authors made a significant contribution to the work reported, whether that is in the conception, study design, execution, acquisition of data, analysis and interpretation, or in all these areas; took part in drafting, revising or critically reviewing the article; gave final approval of the version to be published; have agreed on the journal to which the article has been submitted; and agree to be accountable for all aspects of the work.

\section{Disclosure}

NYF reports grants from Motchida Pharmaceuticals, outside the submitted work, and received research support from Otsuka Pharmaceutical Co., Ltd.; Mochida 
Pharmaceutical Co., Ltd.; Dainippon Sumitomo Pharmaceutical Co.; and MSD Co. YO reports grants from Eisai Co., Ltd., outside the submitted work and received research support from Eisai Co., Ltd. KS has received research support from Novartis Pharma K.K.; Dainippon Sumitomo Pharma Co.; Astellas Pharma, Inc.; Eisai Co., Ltd.; Pfizer, Inc.; Otsuka Pharmaceutical Co., Ltd.; and Daiichi Sankyo Co. and honoraria from Eisai Co., Ltd.; Meiji Seika Pharma Co., Ltd.; Janssen Pharmaceutical K.K.; Sumitomo Dainippon Pharma Co., Ltd.; Eli Lilly Japan K.K.; and Pfizer, Inc. The funders did not have any role in data collection or in the study design, analysis, decision to publish, or preparation of the manuscript. The authors report no other potential conflicts of interest for this work.

\section{References}

1. Hjorthøj C, Stürup AE, McGrath JJ, Nordentoft M. Years of potential life lost and life expectancy in schizophrenia: a systematic review and meta-analysis. Lancet Psychiatry. 2017;4(4):295-301. doi:10.1016/ S2215-0366(17)30078-0

2. Olfson M, Gerhard T, Huang C, Crystal S, Stroup TS. Premature mortality among adults with schizophrenia in the United States. JAMA Psychiatry. 2015;72(12):1172-1181. doi:10.1001/jamapsychiatry.2015.1737

3. Salvo F, Pariente A, Shakir S, et al. Sudden cardiac and sudden unexpected death related to antipsychotics: A meta-analysis of observational studies. Clin Pharmacol Ther. 2016;99(3):306-314. doi: $10.1002 /$ cpt.250

4. Straus SM, Kors JA, De Bruin ML, et al. Prolonged QTc interval and risk of sudden cardiac death in a population of older adults. $\mathrm{J}$ Am Coll Cardiol. 2006;47(2):362-367. doi:10.1016/j.jacc.2005.08.067

5. Bazet $\mathrm{H}$. An analysis of the time-relations of electrocardiograms. Heart. 1920;7:353-370.

6. Reilly JG, Ayis SA, Ferrier IN, Jones SJ, Thomas SH. QTc-interval abnormalities and psychotropic drug therapy in psychiatric patients. Lancet. 2000;355(9209):1048-1052. doi:10.1016/S0140-6736(00) 02035-3

7. Ozeki Y, Fujii K, Kurimoto N, et al. QTc prolongation and antipsychotic medications in a sample of 1017 patients with schizophrenia. Prog Neuropsychopharmacol Biol Psychiatry. 2010;34(2):401-405. doi:10.1016/j.pnpbp.2010.01.008

8. ICH steering committee. ICH harmonized tripartite guideline. The clinical evaluation of QT/QTC interval prolongation and proarrythmic potential for non-antiarrhythmic drugs E14; 2005. Available from: https://database.ich.org/sites/default/files/E14_Guideline.pdf. Accessed October, 102020.

9. Browne KF, Prystowsky E, Heger JJ, Chilson DA, Zipes DP. Prolongation of the Q-T interval in man during sleep. $\mathrm{Am}$ J Cardiol. 1983;52(1):55-59. doi:10.1016/0002-9149(83)90068-1

10. Watanabe J, Suzuki Y, Fukui N, et al. Increased risk of antipsychotic-related QT prolongation during nighttime: a 24-hour holter electrocardiogram recording study. J Clin Psychopharmacol. 2012;32(1):18-22. doi:10.1097/JCP.0b013e31823f6f21

11. Cowan JC, Yusoff K, Moore M, et al. Importance of lead selection in QT interval measurement. Am J Cardiol. 1988;61(1):83-87. doi:10.1016/0002-9149(88)91309-4

12. Higham PD, Furniss SS, Campbell RW. QT dispersion and components of the QT interval in ischaemia and infarction. Br Heart $J$. 1995;73(1):32-36. doi:10.1136/hrt.73.1.32
13. Lubinski A, Lewicka-Nowak E, Kempa M, Baczynska AM, Romanowska I, Swiatecka G. New insight into repolarization abnormalities in patients with congenital long QT syndrome: the increased transmural dispersion of repolarization. Pacing Clin Electrophysiol. 1998;21(1 Pt 2):172-175. doi:10.1111/j.1540-8159.1998.tb01083.x

14. Gupta P, Patel C, Patel H, et al. T (p-e)/QT ratio as an index of arrhythmogenesis. J Electrocardiol. 2008;41(6):567-574. doi:10. 1016/j.jelectrocard.2008.07.016

15. Zabel M, Portnoy S, Franz MR. Electrocardiographic indexes of dispersion of ventricular repolarization: an isolated heart validation study. J Am Coll Cardiol. 1995;25(3):746-752. doi:10.1016/07351097(94)00446-W

16. Higham PD, Campbell RW. QT dispersion. Br Heart J. 1994;71 (6):508-510. doi:10.1136/hrt.71.6.508

17. Okin PM, Devereux RB, Howard BV, Fabsitz RR, Lee ET, Welty TK. Assessment of QT interval and QT dispersion for prediction of all-cause and cardiovascular mortality in American Indians: the Strong Heart Study. Circulation. 2000;101(1):61-66. doi:10.1161/ 01.CIR.101.1.61

18. Day CP, McComb JM, Campbell RW. QT dispersion: an indication of arrhythmia risk in patients with long QT intervals. Br Heart $J$. 1990;63(6):342-344. doi:10.1136/hrt.63.6.342

19. Molnar J, Somberg JC. QT dispersion: still a useful measurement. Cardiology. 2009;112(3):165-167. doi:10.1159/000147949

20. Yamaguchi M, Shimizu M, Ino H, et al. T wave peak-to-end interval and QT dispersion in acquired long QT syndrome: a new index for arrhythmogenicity. Clin Sci. 2003;105(6):671-676. doi:10.1042/ CS20030010

21. Castro-Torres Y, Carmona-Puerta R, Katholi RE. Ventricular repolarization markers for predicting malignant arrhythmias in clinical practice. World J Clin Cases. 2015;3(8):705-720. doi:10.12998/ wjcc.v3.i8.705

22. Zhao X, Xie Z, Chu Y, et al. Association between Tp-e/QT ratio and prognosis in patients undergoing primary percutaneous coronary intervention for ST-segment elevation myocardial infarction. Clin Cardiol. 2012;35(9):559-564. doi:10.1002/clc.22022

23. Ucar FM, Ozturk C, Yilmaztepe MA. Evaluation of Tp-e interval, $\mathrm{Tp}$-e/QT ratio and Tp-e/QTc ratio in patients with acute myocarditis. BMC Cardiovasc Disord. 2019;19(1):232. doi:10.1186/s12872-019$1207-\mathrm{z}$

24. Okayasu H, Ozeki Y, Fujii K, et al. Investigation of the proarrhythmic effects of antidepressants according to QT interval, QT dispersion and $\mathrm{T}$ wave peak-to-end interval in the clinical setting. Psychiatry Investig. 2019;16(2):159-166.

25. Charbit B, Samain E, Merckx P, Funck-Brentano C. QT interval measurement: evaluation of automatic QTc measurement and new simple method to calculate and interpret corrected QT interval. Anesthesiology. 2006;104(2):255-260. doi:10.1097/00000542-200602000-00009

26. Hodges M, Salcrmo D, Erlien D. Bazett's coorection reviewedEvidence that a linear QT correction for heart is better. $J \mathrm{Am}$ Collegue Cardiol. 1983;1:694.

27. Luo S, Michler K, Johnston P, Macfarlane PW. A comparison of commonly used QT correction formulae: the effect of heart rate on the QTc of normal ECGs. J Electrocardiol. 2004;37:Suppl:81-90. doi:10.1016/j.jelectrocard.2004.08.030

28. Haarmark C, Hansen PR, Vedel-Larsen E, et al. The prognostic value of the Tpeak-Tend interval in patients undergoing primary percutaneous coronary intervention for ST-segment elevation myocardial infarction. $J$ Electrocardiol. 2009;42(6):555-560. doi:10.1016/j. jelectrocard.2009.06.009

29. Inada T, Inagaki A. Psychotropic dose equivalence in Japan. Psychiatry Clin Neurosci. 2015;69(8):440-447. doi:10.1111/pcn.12275

30. Roden DM. Predicting drug-induced QT prolongation and torsades de pointes. J Physiol. 2016;594(9):2459-2468. doi:10.1113/JP270526

31. Taylor D, Barnes T, Young A. The Maudsley Prescribing Guidelines in Psychiatry. 13th ed. New York, London: Wiley-Blackwell; 2018. 
32. Aronow WS, Shamliyan TA. Effects of atypical antipsychotic drugs on QT interval in patients with mental disorders. Ann Transl Med. 2018;6(8):147. doi:10.21037/atm.2018.03.17

33. Kuehn BM. FDA warns antipsychotic drugs may be risky for elderly. JAMA. 2005;293(20):2462.

34. Li KJ, Greenstein AP, Delisi LE. Sudden death in schizophrenia. Curr Opin Psychiatry. 2018;31(3):169-175. doi:10.1097/YCO.0000 000000000403

35. Chauhan VS, Krahn AD, Walker BD, Klein GJ, Skanes AC, Yee R. Sex differences in QTc interval and QT dispersion: dynamics during exercise and recovery in healthy subjects. Am Heart J. 2002;144 (5):858-864. doi:10.1067/mhj.2002.125619

36. Haarmark C, Graff C, Andersen MP, et al. Reference values of electrocardiogram repolarization variables in a healthy population. $J$ Electrocardiol. 2010;43(1):31-39. doi:10.1016/j.jelectrocard.2009. 08.001
37. Acciavatti T, Martinotti G, Corbo M, et al. Psychotropic drugs and ventricular repolarisation: the effects on QT interval, T-peak to T-end interval and QT dispersion. J Psychopharmacol. 2017;31(4):453-460. doi:10.1177/0269881116684337

38. Yasui-Furukori N, Shimoda K. Recent trends in antipsychotic polypharmacy in the treatment of schizophrenia. Neuropsychopharmacol Rep. 2020;40:208-210. doi:10.1002/npr2.12127

39. van Noord C, Straus SM, Sturkenboom MC, et al. Psychotropic drugs associated with corrected QT interval prolongation. $J$ Clin Psychopharmacol. 2009;29(1):9-15. doi:10.1097/JCP.0b013e3181 $91 \mathrm{c} 6 \mathrm{a} 8$

40. Timmings PL. Sudden unexpected death in epilepsy: is carbamazepine implicated? Seizure. 1998;7(4):289-291. doi:10.1016/S10591311(98)80020-4

\section{Publish your work in this journal}

Neuropsychiatric Disease and Treatment is an international, peerreviewed journal of clinical therapeutics and pharmacology focusing on concise rapid reporting of clinical or pre-clinical studies on a range of neuropsychiatric and neurological disorders. This journal is indexed on PubMed Central, the 'PsycINFO' database and CAS, and is the official journal of The International Neuropsychiatric Association (INA). The manuscript management system is completely online and includes a very quick and fair peer-review system, which is all easy to use. Visit http://www.dovepress.com/testimonials.php to read real quotes from published authors. 